\title{
Freak Waves Generated by Modulation Instability Under Different Initial Conditions
}

\author{
Jian DING \\ Key Laboratory of Coastal Disaster and Defence (Hohai \\ University), Ministry of Education \\ Nanjing 210098, China \\ Email: dj60hhu@126.com \\ Di WU \\ College of Harbor, Coastal and Offshore Engineering \\ Hohai University \\ Nanjing 210098, China \\ Email:769653558@qq.com
}

\author{
Keren QI \\ China Waterborne Transport Research Institute \\ Beijing 100088, China
}

\author{
Yayi LIU \\ College of Harbor, Coastal and Offshore Engineering \\ Hohai University \\ Nanjing 210098, China
}

\author{
Junwei SU \\ College of Harbor, Coastal and Offshore Engineering \\ Hohai University \\ Nanjing 210098, China
}

\begin{abstract}
Modulation Instability, also called B-F Instability, is widely recognized as the key reason of nonlinear Freak Wave generation. However, the majority of current researches concentrated only on the Most Unstable Condition (MUC for short). In this paper, systematic study covering various occurrence conditions of Modulation Instability has been investigated to discuss the wave train evolution characteristics corresponding to different initial sideband conditions. The results show that under non-MUC, though sidebands grow more slowly and generation of Freak Wave takes longer time, the maximum wave amplitudes tend to increase linearly as the initial ratio of relative spectrum width to wave steepness decreasing. It indicates that compared with the MUC, wave trains corresponding to narrower initial spectrum could produce stronger Freak Waves, also the evolution and characteristic parameters of which demonstrate stronger nonlinearity under non-MUC.
\end{abstract}

Keywords-freak wave; non-MUC; evolution; spectrum parameter; waveform; nonlinearity

\section{INTRODUCTION}

In recent two decades, the existing of Freak Wave as a sudden ocean wave disaster has been acknowledged by the public and aroused wide attention of many researchers.

Among various studies, the generation mechanism of Freak Wave attracts most concerns. It has gained large number of achievements in Modulation Instability which is generally accepted as the key reason of Freak wave generation. However, the majority of current researches concentrated on wave train evolution and characteristic parameters only under the Most Unstable Condition (MUC for short).
As a typical nonlinear mechanism, Modulation Instability, also called Benjamin-Feir Instability or Sideband Instability, is a self-focusing phenomenon imposing disturbances to sideband which is unstable during the evolution of uniform wave train with finite amplitude. After a certain time scale, energy of carrier wave drops down while energy of sideband exponentially raises, that is, energy transfers from carrier wave to sideband. According to the results of Benjamin and Feir, Modulation Instability only works when equation (1) is satisfied.

$$
r_{1}<2 \sqrt{2} \varepsilon_{0}
$$

Where $r_{1} \equiv \Delta k / k_{0}, \Delta k=k_{+}-k_{0}=k_{0}-k_{-}$and $k$ is the wave number.

From previous research, the Freak Wave will appear at modulation peaks along the water wave evolution process. And it is well known that the water wave evolution process will reach the modulation peak within shortest time duration if the initial condition satisfies the MUC. For a given $\varepsilon_{0}$, there should be a corresponding $r_{1}$ (equation (2)) which can induce the fastest sidebands growth with the wave train reaching to the maximum strength.

$$
\left\{\begin{array}{l}
r_{1}=2 \varepsilon_{0} \\
\Delta \theta=\pi / 4
\end{array}\right.
$$

Where $\Delta \theta$ is the phase difference between carrier 
wave and sidebands.

Numerical modulation of Freak Wave generated by Modulation Instability has been carried out under different conditions, including MUC as well as non-MUC. The results show that the maximum wave amplitudes tend to increase linearly as the initial ratio of relative spectrum width to wave steepness decreasing, though sidebands grow more slowly and generation of Freak Wave takes longer time under the non-MUC. Due to the research for Freak Wave focusing more on its maximum wave amplitude, we need to study Freak Wave not only under the MUC, but also pay attention to the evolution and the characteristics of Freak Wave under the non-MUC.

\section{HIGH ORDER SPECTRA(HOS)}

The high-order spectral method (HOS) developed by Dommermuth \& Yue(1987)is applied for direct phaseresolved simulation of nonlinear random wave fields evolution. HOS resolves the phase of a large number $(\mathrm{N})$ of wave modes and accounts for their nonlinear interactions up to an arbitrary high order (M) ncluding broadband nonresonant and resonant interactions up to any specified order. Meanwhile viscous dissipation and wave breaking dissipation are modeled in domain. Due to its high efficiency and accuracy, HOS is an effective approach for long-time and large-space simulation of nonlinear wave-field evolutions. At present, the method has been widely applied in nonlinear water wave hydrodynamics and a variety of applied researches have been carried out.

\section{FREAK WAVE EVOLUTION GENERATED BY MODULATION INSTABILITY}

\section{A. Initial Conditions Of Weakly Modulated Wave Train}

For the narrow band modulated wave train, the initial conditions we used are as following.

$$
\left.\begin{array}{l}
\eta(x, 0)=\eta\left[\varepsilon_{0}, k_{0}\right]+r_{1} a_{0} \cos \left(k_{-} x-\theta_{-}\right)+r_{2} a_{0} \cos \left(k_{+} x-\theta_{+}\right) \\
\phi^{s}(x, 0)=-\phi^{s}\left[\varepsilon_{0}, k_{0}\right]+\frac{r_{1} a_{0}}{\sqrt{k_{-}}} e^{k_{-} \eta} \sin \left(k_{-} x-\theta_{-}\right)+\frac{r_{2} a_{0}}{\sqrt{k_{+}}} e^{k_{+} \eta} \sin \left(k_{+} x-\theta_{+}\right)
\end{array}\right\}(3)
$$

where $\eta(x, 0)$ and $\varphi^{s}(x, 0)$ are respectively the freesurface elevation and potential of a right-going Stokes wave of steepness $\varepsilon_{0}$ and wave number $k_{0}$. Here, $\varepsilon_{0}=k_{0} a_{0}$ and $a_{0}$ is the surface elevation of carrier wave. $k \pm=k_{0} \pm \Delta k$ and $\theta_{ \pm}$are wave numbers and phases of sideband respectively. Those parameters are chosen as the most unstable conditions in the initial period. The initial wave relative amplitude spectrum and wave surface for 0.05 are shown in Fig. 1. For all the cases, the calculation domain is 100. The parameters for HOS are $\mathrm{M}=4, \mathrm{~N}=4096, \mathrm{~T} / \mathrm{dt}=64$, here $\mathrm{dt}$ is the time step for simulation.

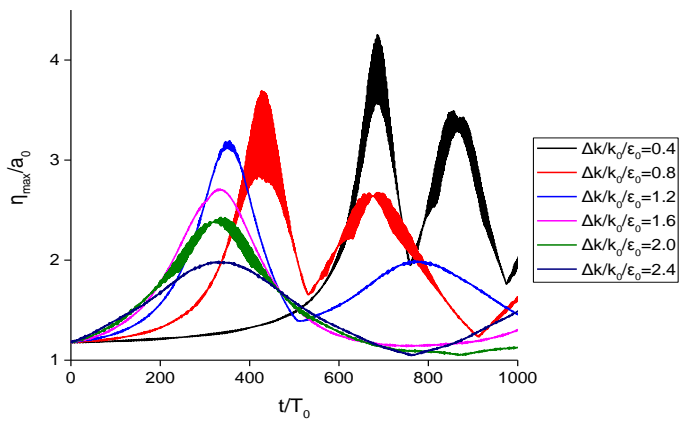

Figure 1. Wave amplitude evolutions of different initial sideband loading location

\section{B. Evolution of the First Modulated Maximum Freak Wave}

Modulation instability, can also be called Benjamin and Feir instability, has been observed and studied in numerous physical fields including water waves, plasma waves, laser beams, and electromagnetic transmission lines. Benjamin and Feir found that the unstable sideband components would grow exponentially with a time rate which depends on the dimensionless frequency difference between the carrier wave and unstable sideband and the initial wave steepness.

According to results of Benjamin and Feir, Modulation Instability works only when $r_{1}<2 \sqrt{2} \varepsilon_{0}$. For a given $\varepsilon_{0}$, there should be a corresponding $r_{1}$ which can induce the fastest sidebands growth, and this can be called the most unstable condition (MUC), which is the emphasis of many current studies. Taking $\varepsilon_{0}=0.05$ the case for example, the results are shown in Fig. 1, which is the evolution process of wave surface with different initial sideband conditions within modulation instability range. It is obvious that the wave train with $\Delta k / k_{0}=2 \varepsilon_{0}$ is the fastest that reaches the modulation peak, while the maximum wave amplitudes tend to increase linearly as the initial ratio of relative spectrum width to wave steepness decreasing, namely, $\Delta k / k_{0}=1.6 \varepsilon_{0}, \Delta k / k_{0}=1.2 \varepsilon_{0}, \Delta k / k_{0}=0.8 \varepsilon_{0}$ and $\Delta k / k_{0}=0.4 \varepsilon_{0}$. The maximum wave amplitude is four times to the initial value though it takes longer time for evolution.

As shown in Fig. 2, the evolution process of wave surface with different initial sideband conditions are put together to compare. It is clear to see that the modulation instability only works when the initial sideband condition satisfying a certain range. Also, the freak wave under the MUC turns out not the largest one, while the maximum wave amplitudes caused by modulation instability tend to grow larger as the initial ratio of relative spectrum width to wave steepness decreasing. Relationship between maximum wave amplitude and initial sideband loading location parameter $\mathrm{xxx}$ is given in equation (4), which the correlation coefficient is up to 0.98 . 
$\eta_{\max }=f\left(\Delta k / k_{0} / \varepsilon_{0}\right)=\left\{\begin{array}{lr}-1.26\left(\Delta k / k_{0} / \varepsilon_{0}\right)+4.94, & 0<\Delta k / k_{0} / \varepsilon_{0} \leq 2 \sqrt{2} \\ 1.27, & \Delta k / k_{0} / \varepsilon_{0}>2 \sqrt{2}\end{array}\right.$

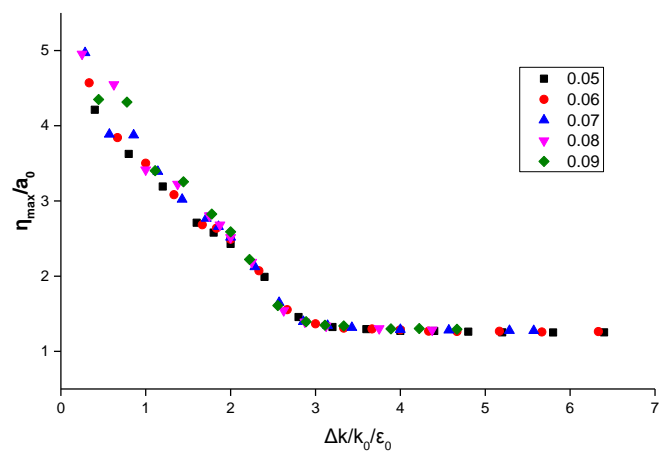

In order to analyze the evolution of the first modulated freak wave, our study concentrates mainly on frequency spectrum, spatial wave field and the variation of the main sidebands. Taking for example, $\Delta k=10$ is the most unstable sideband in the case. As the initial sideband reaching closer to the carrier wave, namely, $\Delta k=10$ $\Delta k=6, \Delta k=2$, the frequency spectrum, spatial wave field and the variation of the main sidebands are shown in Fig. 3. The simulation results indicate that, as the initial sideband reaching closer to the carrier wave, the interactions between the two could intimate more waves participating into the energy exchange, the state of which is unable to recover. Also, it takes longer time to reach to the first modulation state, while the corresponding freak wave height is larger and the asymmetry of waveform is more obvious.

Figure 2. Maximum wave amplitudes of initial wave steepness with different initial
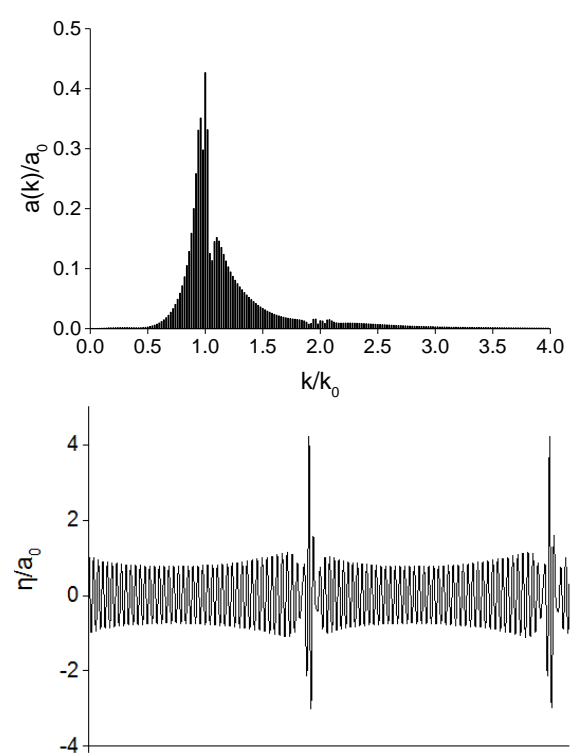

Spatial wave field



(a)
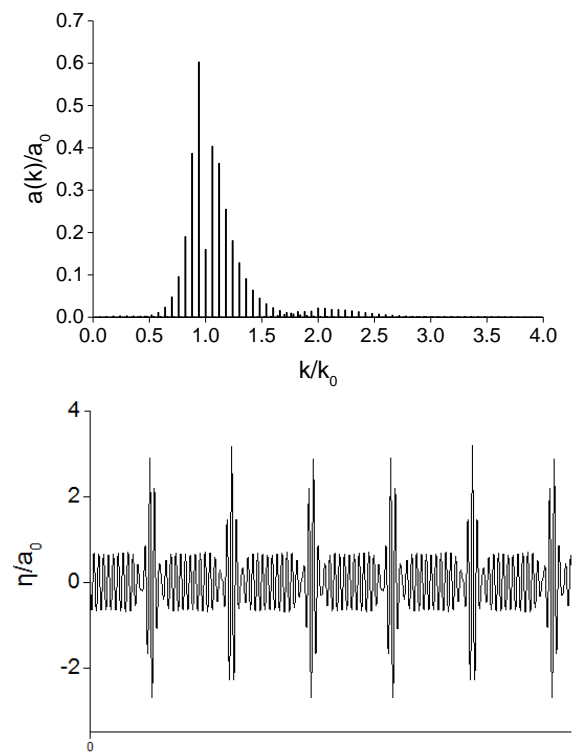

Spatial wave field

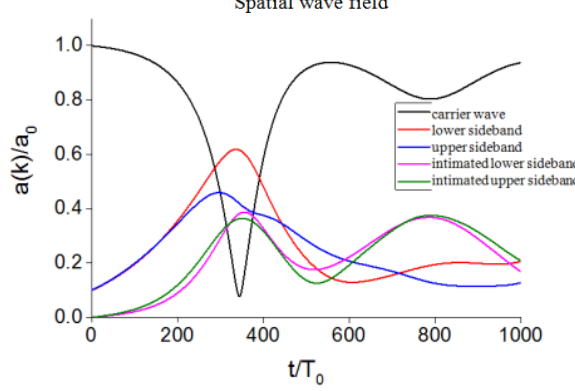

(b)
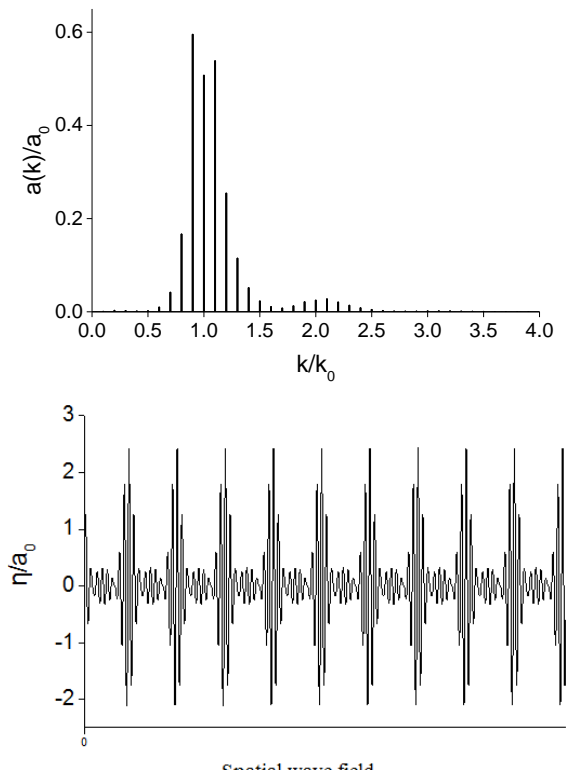

Spatial wave field

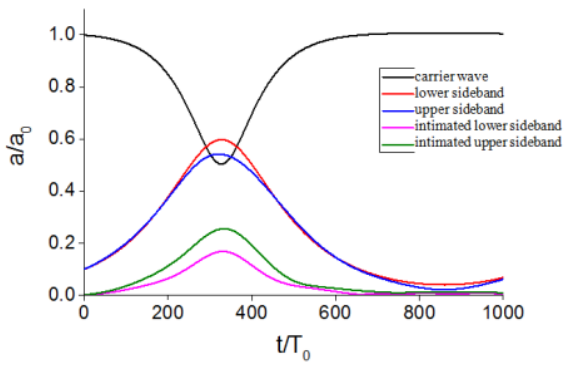

(c)

Figure 3. Frequency spectrum, spatial wave surface and evolutions of carrier wave and main sidebands at the first modulation time of different initial sideband loading location (a) (b) (c) 


\section{Spectral Parameters}

In order to further explore the influence of the initial sideband loading location to the wave field evolution, simulations under different conditions are carried out to analyze the spectral width and $N_{g}$. In this paper, equation (5) is adopted to calculate the spectral width, which is relatively simple.

$$
\begin{gathered}
\mathrm{P}=\frac{S\left(\omega_{0}\right) \omega_{0}}{m_{0}} \\
B=1 / P
\end{gathered}
$$

Where $m_{0}$ is 0 -order spectral moment, $\omega_{0}$ is spectral peak frequency. $\mathrm{P}$ is the spectral steepness describing the wind wave development, which represents narrower energy distribution with larger value. $\mathrm{B}=1 / \mathrm{P}, \mathrm{B}$ is the spectral width. As shown in Fig. 4, the spectral width of different initial sideband conditions that satisfying the modulation instability condition are compared. It is clear to see that the spectral width with different initial wave steepness tends to grow larger as the sideband loading location closer to the carrier wave, which means its corresponding energy distribution is broader. In the modulation instability working range, there is a good exponential relationship between the wave spectral width $\mathrm{B}$ and the initial sideband loading location parameter $\Delta k / k_{0} / \varepsilon_{0}$.

$N_{g}$ is the ratio of wave numbers that $5 \%$ greater than the initial wave amplitude to the whole wave number in the frequency domain. The definition is given in equation(6).

$$
\begin{gathered}
N_{g}=\sum n_{i} / N \\
n_{i}=\left\{\begin{array}{rr}
1, & \frac{a\left(n_{i}\right)}{a_{0}} \geq 5 \% \\
0, & \text { else }
\end{array}\right.
\end{gathered}
$$

Where $n_{i}$ is the wave of the $i^{\text {th }}$ frequency, $\mathrm{N}$ is the number of frequencies in the calculation range and $a_{i}$ is the wave amplitude of the $i^{\text {th }}$ frequency. From the case of $\varepsilon_{0}=0.05,0.06,0.07,0.08,0.09$ in Fig. 5, it is obvious that $N_{g}$ increases as the initial sideband loading location closer to the carrier wave, which means there are more waves of different frequencies imitated out in the wave field at the first modulation moment.

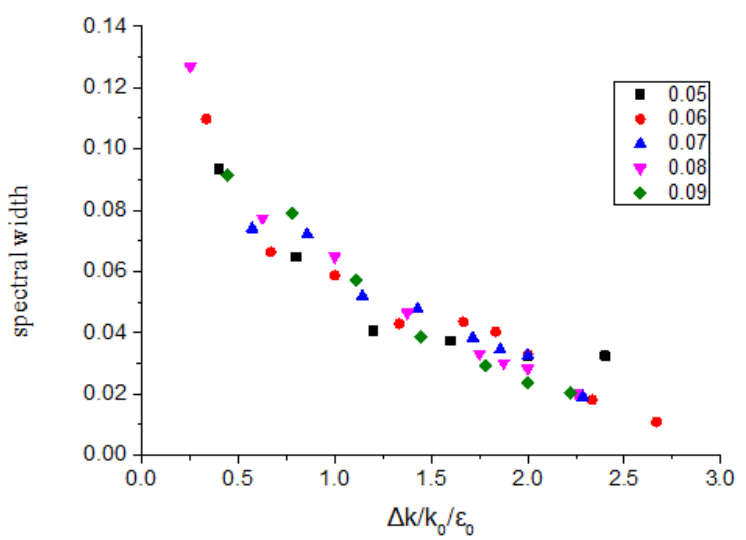

Figure 4. Spectral width of initial wave steepness with different initial sideband loading location

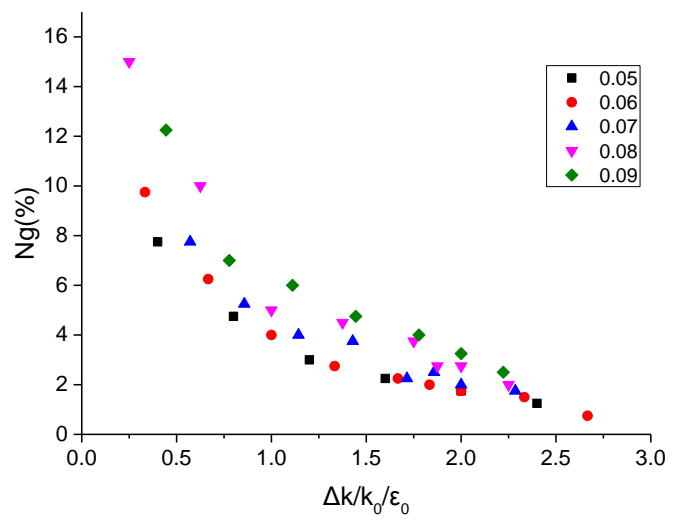

Figure 5. $\quad N_{g}$ of initial wave steepness with different initial sideband loading location

\section{Characteristics of Modulated Freak Wave}

The characteristics of freak wave generated by modulation instability are studied in this paper by analyzing its waveform parameters, local wave steepness and freak degree. Waveform parameters include horizontal symmetry and vertical symmetry. The definition of horizontal symmetry is the ratio of wave crest to wave height, namely $\eta_{c} / H$. Vertical symmetry is the ratio of wave trough duration to wave crest duration, namely $T_{t} / T_{c}$. The results of horizontal symmetry and vertical symmetry of the case from $\varepsilon_{0}=0.05$ to $\varepsilon_{0}=0.09$ are given in Fig. 6 . 



Figure 6. Horizontal symmetry and vertical symmetry of initial wave steepness with different initial sideband loading location

From Fig. 7, it is clear to see that there are significant differences in the freak wave characteristics due to the initial sideband loading location. As the initial sideband located closer to the carrier wave, for horizontal symmetry, wave crest proportion increases with steeper wave crest and smoother wave trough. For vertical symmetry, there is larger difference in the duration of wave crest and wave trough with shorter wave crest duration and longer wave trough duration.

Local wave steepness and freak degree represent the nonlinear degree of freak wave. The results of local wave steepness and freak degree of the case from $\varepsilon_{0}=0.05$ to $\varepsilon_{0}=0.09$ are given in Fig. 7 .

Figure 7. Local wave steepness and freak degree of initial wave steepness with different initial sideband loading location

The variation of local wave steepness and freak degree are relatively similar as the initial sideband loading location changes. As the initial sideband located closer to the carrier wave, local wave steepness of different initial wave steepness tends to grow and the trend is more significant with larger initial wave steepness. For freak degree, its variation is inconspicuous with small initial wave steepness, while increases with the sideband loading location closer to the carrier wave as the initial wave steepness is larger.

The waveform characteristics, local wave steepness and freak degree represent the nonlinear degree of freak wave. In all, as the initial sideband located closer to the carrier wave, the nonlinear degree of freak wave tends to increase, which is more significant when the initial wave steepness is larger.

\section{CONCLUSION}

In this paper, simulations of Stokes wave train evolution corresponding to different initial sideband loading location satisfying modulation instability are carried out to analyze the evolution and characteristic variation. As the initial sideband reaching closer to the carrier wave, the interactions between the two intimate more waves participating into the energy exchange, the state of which is unable to recover. 
Also, it takes longer time to reach to the first modulation state, while the corresponding freak wave height is larger and the asymmetry of waveform is more obvious. Besides, comparing to the most unstable condition, under non-MUC, freak wave generated by modulation instability tends to show stronger nonlinearity, which is more obvious with larger initial wave steepness.

\section{REFERENCES}

[1] Benjamin T B, Hasselmann K. Instability of periodic wavetrains in nonlinear dispersive systems[C]. Proceedings of the Royal Society of London A: Mathematical, Physical and Engineering Sciences. The Royal Society, 1967, 299(1456): 59-76.

[2] Benjamin T B, Feir J E. The disintegration of wave trains on deep water Part 1. Theory[J]. Journal of Fluid Mechanics, 1967, 27(03): 417-430.

[3] Tao A, Zheng J, MeeMee S, et al. The Most Unstable Conditions of Modulation Instability[J]. Journal of Applied Mathematics, 2012.
[4] Tao A, Mee M S. Re-study on recurrence period of Stokes wave train with High Order Spectral method[J]. China Ocean Engineering, 2011, 25(4): 679-686.Dommermuth D G, Yue D K P. The nonlinear threedimensional waves generated by a moving surface disturbance[C]. Proceedings of the 17th Symposium on Naval Hydrodynamics. 1988: 59-71.

[5] Dommermuth D G, Yue D K P. A high-order spectral method for the study of nonlinear gravity waves[J]. Journal of Fluid Mechanics, 1987, 184: 267-288.

[6] West B J, Brueckner K A, Janda R S, et al. A new numerical method for surface hydrodynamics[J]. Journal of Geophysical Research: Oceans (1978-2012), 1987, 92(C11): 11803-11824.

[7] Wu G. Direct simulation and deterministic prediction of large-scale nonlinear ocean wave-field[D]. Massachusetts Institute of Technology, 2004.

[8] Zhang J, Hong K, Yue D K P. Effects of wavelength ratio on wave modelling[J]. Journal of Fluid Mechanics, 1993, 248: 107-127.

[9] Liu Y, Yue D K P. On generalized Bragg scattering of surface waves by bottom ripples[J]. Journal of Fluid Mechanics, 1998, 356: 297-326. 